\title{
DETERMINATION OF THE THICKNESS OF A RESISTIVE MATERIAL LAYER IN A FINITE VOLUME CONDUCTOR USING FOCUSED IMPEDANCE METHOD (FIM) - A SIMULATION STUDY
}

\author{
Golam Dastegir Al-Quaderi ${ }^{1 *}$, Sayed Parvez Ahmed ${ }^{2}$, K Siddique-e Rabbani ${ }^{3}$ \\ ${ }^{1}$ Department of Physics, University of Dhaka, Dhaka, Bangladesh. \\ ${ }^{2}$ Department of Physics, Jahangir Nagar University, Savar, Bangladesh. \\ ${ }^{3}$ Department Biomedical Physics \& Technology, University of Dhaka, Dhaka, Bangladesh. \\ *Corresponding author: dastegir@gmail.com
}

Received: 08 June 2014, Accepted: 15 July 2014

\begin{abstract}
Focused Impedance Method (FIM) is an innovative and relatively new electrical impedance measurement technique that allows localized measurement of the properties of electrically conducting materials in a volume conductor such as the human body using simple measurement setups. In a previous work, FIM, with dual electrode separation, was used to determine thicknesses of subcutaneous fat layers through experimental study on phantoms and human subjects. The present work, carried out using COMSOL Multiphysics software package, is a finite element simulation study for a similar target object that verifies and extends the results of the previous experimental work. A rectangular box of different heights containing a material of uniform conductivity such as saline together with internally embedded layers of resistive materials of different thicknesses were used for this study. The measured transfer impedance in the FIM method showed marked change with the variation of the thickness of the resistive layer and with electrode separation, showing a point of maximum curvature in the latter. We obtained a calibration curve for the thickness from the electrode separation of this point. This allowed a unique method for the determination of thickness of embedded resistive layers which is more general than what had been done in the previous experimental work. This work will help standardize the application of 4-electrode FIM for determination of the thickness of less-conducting material layer in any finite volume, including determination of fat layer thickness, etc.
\end{abstract}

Keywords: Electrical Impedance, focused impedance method (FIM), finite element model, simulation, thickness of resistive layer

\section{INTRODUCTION}

Electrical impedance measurement techniques provide a tool for diagnosis of tissue characteristics (Schwan 1957) and hence are of medical importance. This is because biological materials have in general both dielectric and conductive properties (Pethig 1979). Besides serving diagnostic purposes, electrical impedance can also be used to get information about the bulk properties like size of objects within a volume conductor (Smulders and Oosterom 1992, Kadir et al. 2013, Brown et al. 1994), thickness of resistive layers (of both biological (Surovy et al. 2012, Haowlader et al. 2010, Birgersson et al. 2012) and inanimate materials (Weinstein 2003)), or the position of an inhomogeneity within a bulk conductor. Besides being non-invasive, electrical impedance measurement also has the advantage of being relatively low cost, simple and ready-to-use technique as compared to other diagnostic techniques like the MRI or CT scans which require sophisticated and elaborate instrumentation. Biological tissues possess two very important passive electrical properties: electrical conductivity due to free charge carriers (ions etc.) and dielectric properties like relative permittivity due to bound charge densities. An alternating current flow through such a material thus is governed by the distribution of both permittivity and conductivity within it. Variation of one or both thus affects the transfer impedance measured between appropriate sets of electrodes. 
A volume conductor having an embedded layer of relatively higher or lower resistive material than that of the bulk occurs in many practical applications and in natural settings. Subcutaneous fat and adipose tissue has a larger resistivity compared to the inner body tissue. Electrical impedance measurement from the outside will be dependent on the distribution of relative electrical conductivity and permittivity at different positions inside the volume and hence can in principle determine the thickness of the embedded layer if the depth and the impedivity of the embedded layer are known. Determination of the thicknesses of such embedded layers is often required in medical and industrial applications. In all such cases, electrical impedance measurement techniques could easily be applied.

Scharfetter et al, 2001 reported good correlation $\left(r^{2}=0.99\right)$ between the impedance measured across the waist of a number of subjects using Tetrapolar Impedance Measurement (TPIM) technique and the thickness of the subcutaneous fat layer obtained using MRI. This TPIM used a linear array of four electrodes with current driven through the outer pair (source electrodes) and potential measured across the inner pair (receive electrodes), aiming to get localized transfer impedance (referred to as simply impedance in the whole of the following text) of the central zone, between the two receive electrodes. This arrangement has a wide zone of sensitivity so that contributions from tissues at locations other than the desired zone affect the impedance value as well. Besides, there are also zones with considerable values of negative sensitivity which again affect the measured impedance. Furthermore, variation of the electrode separation will affect the impedance value significantly and in a nontrivial way. To investigate this last effect, our extended group had used the linear TPIM configuration as above but with varying electrode separations (Haowlader et al 2010). For this study the electrodes were vertical pins piercing a thick polystyrene foam block which was made to float on saline in a rectangular tank simulating a simplified biological volume conductor. The study investigated electrical impedance at low frequencies when the dielectric properties do not play a significant role. Flattened heads of the electrodes touched the saline surface underneath. Spongy plastic foam sheets, placed underneath the polystyrene foam block, were pressed into the saline, to simulate the fat layer (higher resistivity than underlying saline). Measurements were performed for different thicknesses of the spongy foam (simulated fat). Through preliminary measurements it appeared that it may be possible to develop a technique for determination of the thickness of such resistive layers through variation of electrode separations of TPIM and the following systematic study was performed. The receive electrode separation was kept fixed and the source electrode separation was increased. The measured impedance remained almost constant at low values of the source electrode separation. As the source electrode separation increased, the impedance fell sharply, showing a knee. This decrease continued until a saturation value reached. The knee at which the impedance value fell sharply increased for increasing thickness of immersed foam layer. This suggested a way to assess the thickness of the resistive foam layer from the position of the knee in the impedance curve. However, the knee occurred at low electrode separation which limited the use of the method for resistive layers beyond a certain thickness.

Inspired by the success of the above work, another team of our extended group used a new technique, Focused Impedance Method (FIM) for the measurement of thickness of fat layer, through a phantom study and measurements on human subjects (Surovy et al. 2012). FIM is a relatively new impedance measurement technique developed by our extended group at the University of Dhaka, Bangladesh (Rabbani et al 1999, Rabbani and Karal 2008 ). In this method impedance is measured as in TPIM but in two concentric and mutually perpendicular directions, which gives an enhanced sensitivity in the central region; this also reduces the magnitude of the contributions of the zones with negative sensitivity (Islam et al. 2010). 


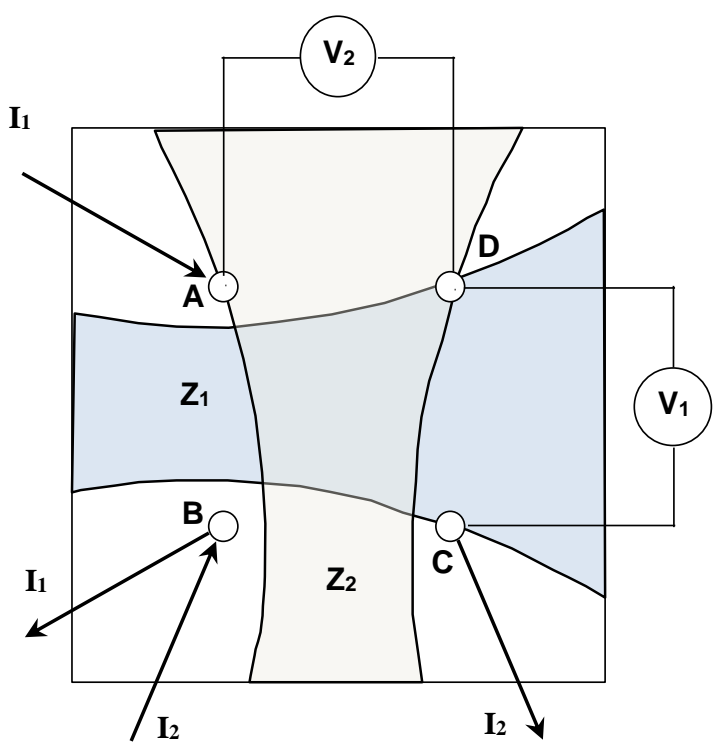

Figure-1: Basic concept of 4-electrode FIM or FIM-4. For simplicity we have taken $\mathrm{I}_{1}=\mathrm{I}_{2}$

There are three versions of FIM, the first two are essentially based on the linear TPIM configuration; one using 8 electrodes while the other using 6 electrodes (Rabbani et al. 1999, Islam et al. 2010). The third version uses 4 electrodes, placed at the corners of a square region as shown in Figure 1 (Rabbani and Karal, 2008). This version has been shown to have a better focusing effect and reduced contribution due to zones of negative sensitivity compared to other versions of the FIM (Islam et al, 2010 ). In this method (Figure 1) an alternating current of constant amplitude $I_{1}$ is passed between electrodes 1 and 2 and potential difference between electrodes 3 and 4 is measured. The transfer impedance $Z_{1}$ is defined as the ratio of this voltage difference to the current. This is essentially square TPIM with sensitive zone shown as the shaded region in the figure. Next another alternating current of constant amplitude $I_{2}$ (which may be equal to $I_{1}$ ) is passed between the electrodes 2 and 3 and potential difference is measured between electrodes 1 and 4 . The ratio of this voltage difference to the current gives transfer impedance $\mathrm{Z}_{2}$. The FIM value is defined simply as the sum of the two transfer impedances $\left(Z_{1}+Z_{2}\right)$. The sensitive zone of the combined measurement have a common region from where we expect an enhanced contribution to the FIM value, and hence a better focusing. Surovy et al. used this 4-electrode version of FIM (FIM-4) for three electrode separations. The FIM values, with respect to the electrode separation for different thicknesses of the simulated fat layers were plotted, all of which showed gradual decrease. The plots however had different 'slopes' for different thicknesses of resistive layers, which offered a method to obtain an unknown thickness of resistive layer. The height of the saline was kept at $6 \mathrm{~cm}$ for all thickness of the resistive layers.

The present work firstly takes up a finite element simulation study of the work done by Surovy et al. and extends it further with multiple electrode separations and thicknesses (in small steps) of the resistive layers. Subsequently, it comes up with an alternative technique to determine the thickness of the resistive layers embedded inside a volume conductor near its surface.

\section{METHODS}

Finite element simulation is a standard method of solving boundary value problems in physics, which divides a region into small elements using a geometric mesh and performs solutions to appropriate partial differential equations at each of these mesh points. It is often used to study physical phenomena where experimental studies have limitations and/or are difficult to perform. Furthermore, 
it offers variation of parameters of the study in small steps, as mentioned above, and exploration of extreme cases. In the present work we used the AC/DC module of the software package COMSOL Multiphysics, which used tetrahedral elements.

Model Description: In the present simulation study a rectangular box of base area $12 \mathrm{~cm}$ x $12 \mathrm{~cm}$ was used. The height $\mathrm{H}$ could be varied for the experiment. Four electrodes for an FIM-4 configuration were placed centrally and symmetrically in the base (xy-plane) with the origin taken at this center. The box contained an electrically conductive material, taken to be saline in this study, which simulated a simple conductive body tissue, appropriate at low frequencies $(<50 \mathrm{kHz})$. A single layer of resistive material, with resistivity higher than that of the background was introduced inside the box, just touching the base and covering the whole area. The thickness of the resistive layer was increased in $1 \mathrm{~mm}$ steps up to $1 \mathrm{~mm}$ less than the height $\mathrm{H}$ of the rectangular box. Again, the height $\mathrm{H}$ of the box was also varied $(6,8,10,12 \mathrm{~cm})$ for exploring effect of $\mathrm{H}$, if any. The electrode separation was varied from a modest $1 \mathrm{~cm}$ to almost that of the lateral dimension of the box $(12 \mathrm{~cm})$. The electrodes, cylindrical in shape with diameters of $0.25 \mathrm{~cm}$, were taken to be made up of copper with their face in the base (not protruding inside the box), the rest being outside. The material properties of the saline, resistive layer and electrodes are shown in Table 1.

Table 1: Material properties of saline, resistive layers and electrode material

\begin{tabular}{|c|c|c|}
\hline Type of the material & Relative Permittivity $\epsilon_{\mathrm{r}}$ & Conductivity $\sigma\left(\mathrm{S} \mathrm{m}^{-1}\right)$ \\
\hline Saline (simulated body tissue) & 100.0 & 1.0 \\
\hline Resistive layers (simulated fat) & 600.0 & $40.0 \times 10^{-3}$ \\
\hline Electrodes $(\mathrm{Cu})$ & 1.0 & $5.98 \times 10^{7}$ \\
\hline
\end{tabular}

The models in COMSOL had electrical insulation at all the external boundary (since current is not supposed to flow out of the box) except at the electrodes where copper was used. At the boundary between the two domains, that of the resistive material and of the saline, current could pass and current conservation was imposed in all the domains. The number of vertex elements used in the Finite Element Method (FEM) study was 44 for models with resistive layers and 40 for the models with saline only, respectively. The cylindrical electrodes were automatically divided into eight parts by the solver. The mesh for the finite element method was chosen to be 'physics-controlled' finer mesh (as available as a command in COMSOL), which automatically takes more mesh points at regions of greater current density. For our model the typical number of tetrahedral elements was of the order of 63,000 .

For FIM, we need constant current drives in two perpendicular directions through two paircombinations of source electrodes. We took the same current amplitude, $I_{1}=I_{2}=1$ Amp, through both the drive pairs. The corresponding receive electrode pairs were used as probes for the electric potentials which gave the values of the two transfer impedances in an arbitrary unit since the voltage is proportional to the impedance if the current is constant. We calculated the absolute value of the sum of the two transfer impedances which is the FIM value as described before. 


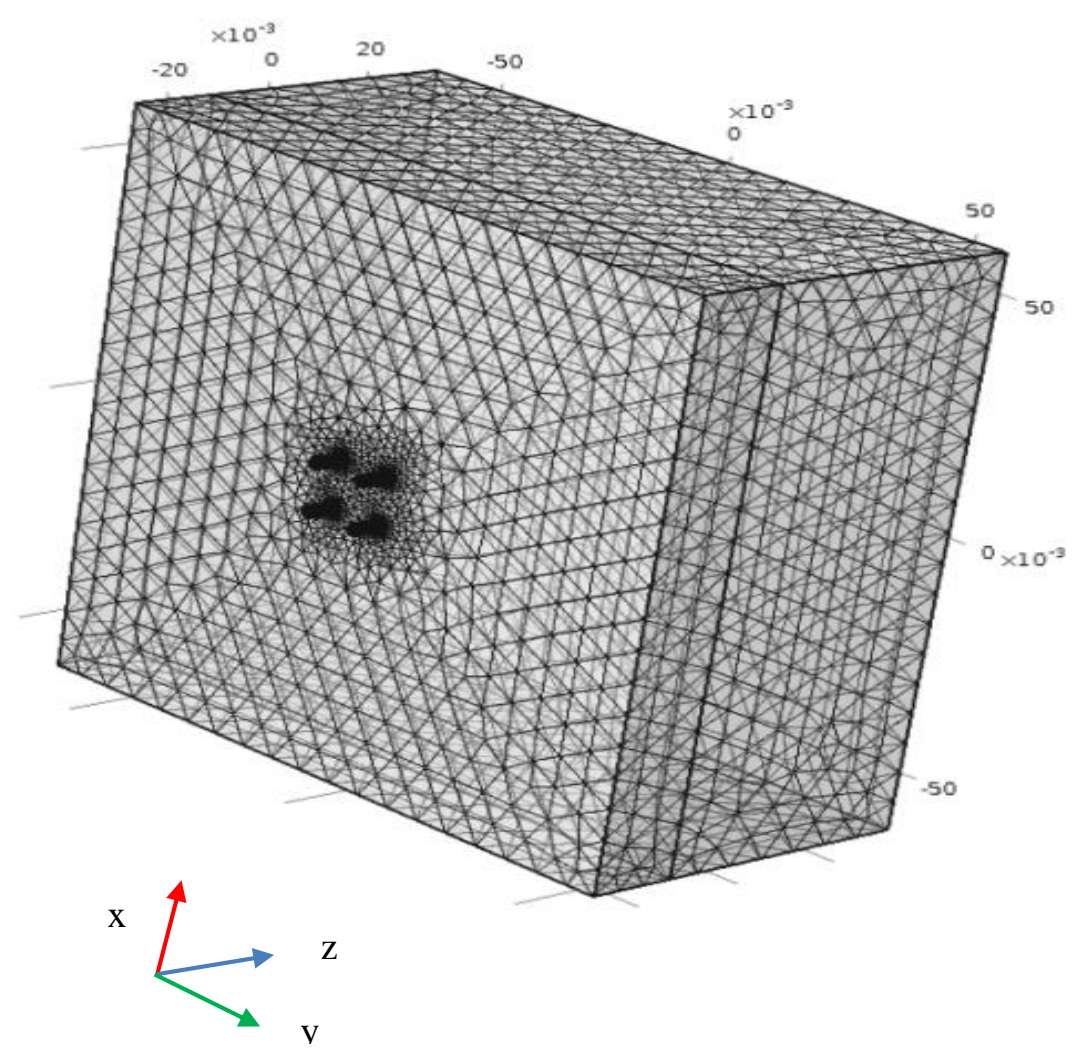

Figure-2: Mesh generated using 'physics controlled' finer mesh option of COMSOL.

Simulation set-up. The boundary value problem is defined in terms of a set of partial differential equations which are then solved using Finite Element Method (FEM) with specified boundary conditions. An alternating current of specified frequency and constant amplitude is input at the source terminals which results in alternating potentials having both real and imaginary parts. In the COMSOL Multiphysics software we have used the Electric Current interface with stationary solvers in the frequency domain. The differential equations solved are

$$
\begin{gathered}
\vec{\nabla} \cdot \vec{J}=Q \\
\vec{J}=\sigma \vec{E}+j \omega \epsilon_{0} \epsilon_{r} \vec{E}+\vec{J}_{e} \\
\vec{E}=-\vec{\nabla} V
\end{gathered}
$$

with the electric insulation equation at the external surfaces (zero perpendicular component):

$$
\hat{\mathrm{n}} . \vec{J}=0
$$

where $\vec{J}$ is the electric current density, $Q$ is net charge within any volume, $\vec{E}$ is the electric field, $\sigma$ is the conductivity, $\omega$ is the angular frequency of the signal, $\epsilon_{0}$ is the permittivity of free space, $\epsilon_{r}$ is the relative permittivity of the material, $\vec{J}_{e}$ is the external electric current density (if any) and $V$ is the electric potential at any point. Throughout our simulations, we have taken the linear frequency to be $10 \mathrm{kHz}$.

Studies Performed: The transfer impedance in FIM-4 (i.e. the FIM value) will in general depend on (i) the electrode separation $\left(\delta_{e l}\right)$, (ii) thickness of the resistive layer $(t)$ and (iii) the size of the box (length=width $=L$ and height $=H$ ), given the material properties kept constant. It needs to be mentioned that for a fixed $H$, as $t$ increases, the thickness of the saline volume $(=H-t)$ decreases. We 
performed several studies in our model to find the effect of these parameters on the transfer impedance. These are outlined below:

(a) Variation of Transfer Impedance with Thickness of resistive layer: The thickness of the resistive layer was varied from $1 \mathrm{~mm}$ to $(H-1) \mathrm{mm}$, where $H$ is the height of the box, kept constant. Four different values of $H$ were used $(H=6 \mathrm{~cm}, 8 \mathrm{~cm}, 10 \mathrm{~cm}$ and $12 \mathrm{~cm})$. The electrode separations $\left(\delta_{e l}\right)$ were taken to be $3.5 \mathrm{~cm}, 5.7 \mathrm{~cm}$ and $8.5 \mathrm{~cm}$.

(b) Variation of Transfer Impedance with Electrode Separation: The transfer impedance was calculated with electrode separations $\left(\delta_{e l}\right)$ ranging from $1 \mathrm{~cm}$ up to $11.7 \mathrm{~cm}$ (just short of the lengths of the sides of the box of $12 \mathrm{~cm}$ ) with an increment of $1 \mathrm{~mm}$ for several fixed thicknesses of the resistive layers $(t=5 \mathrm{~mm}, 10 \mathrm{~mm}, 15 \mathrm{~mm}, 20 \mathrm{~mm}$ and $25 \mathrm{~mm}$ ). The box height was taken to be different but fixed in each series of the thickness.

(c) Variation of the Transfer Impedance with Height of the box: The transfer impedance was calculated with resistive layer of specified thicknesses $(t=5 \mathrm{~mm}, 10 \mathrm{~mm}, 15 \mathrm{~mm}, 20 \mathrm{~mm}$ and $25 \mathrm{~mm})$ and for saline only $(t=0 \mathrm{~mm})$ with different heights of the box $(H=4 \mathrm{~cm}, 6 \mathrm{~cm}, 8 \mathrm{~cm}, 10 \mathrm{~cm}$ and 12 $\mathrm{cm}$ ) and for a fixed $12 \mathrm{~cm}$ as the lengths of the sides of the box. The electrode separations were taken to be fixed at three values $(3.5 \mathrm{~cm}, 5.7 \mathrm{~cm}$ and $8.5 \mathrm{~cm})$.

(d) Variation of the Transfer Impedance with Length or Width of the box: We expect change of the transfer impedance with change of the length or width of the box (length=width $=L$ ) relative to the electrode separation $\left(\delta_{e l}\right)$. We calculated the ratio $r=\delta_{e l} / L$ for this study. Since, the electrode separation was taken to vary from a small value of $10 \mathrm{~mm}$ up to $117 \mathrm{~mm}$ (which is almost equal to the width of the box), the ratio varied from $10 / 120$ to $117 / 120$ i.e. from 0.0833 to 0.975 . The study described in (b) was used to obtain the dependence of the transfer impedance on $L$.

Point of Maximum Curvature for Thickness-Determination; A New Method: From an observation of the variation of transfer impedance with electrode separation for different resistive layer thicknesses ' $t$ ' (study-b), it appeared that the point of maximum curvature varied with ' $t$ ', which could possibly be used to devise a technique for the measurement of ' $t$ '. To remove the dependence on the box height, we used the normalized transfer impedance, $Z_{N}=Z(t) / Z_{0}$, where $Z(t)$ is the transfer impedance with a thickness $t$ of the resistive material and $Z_{0}$ is the transfer impedance with saline only (no resistive layer). The normalized impedance is expected to decrease with increase of electrode separation. The curvature of this plot was calculated at each value of the electrode separation using the well-known formula:

$$
K=\frac{\left|d^{2} y / d x^{2}\right|}{\left(1+\left(\frac{d y}{d x}\right)^{2}\right)^{3 / 2}}
$$

where, $y=Z_{N}$ and $x=t$ for our case. Under the assumption that the position of the point of maximum curvature is a function of the thickness of the resistive layer, a calibration curve is obtained using these two lengths.

\section{RESULTS AND OBSERVATIONS:}

We present below results of the variation of the FIM transfer impedance with respect to different parameters separately. 
(a) Variation of Transfer Impedance with Thickness of the Resistive Layer:

The results of this study are presented in Figure 3 for a box with $H=6 \mathrm{~cm}$. At low values of $t$, the FIM value appears to be almost constant with increasing $t$. Again, for larger $\delta_{e l}$ (electrode separation) this constant value extended to greater values of $t$.

After this value, which we may call the 'first knee thickness', impedance rises in a non-trivial manner. For smaller electrode separations the rise is nonlinear in nature and for larger electrode separations, it becomes increasingly linear. At higher values of the thickness, comparable to the height of the box, we see a sharp increase of the impedance, starting from a thickness value which we may call the 'second knee thickness'.
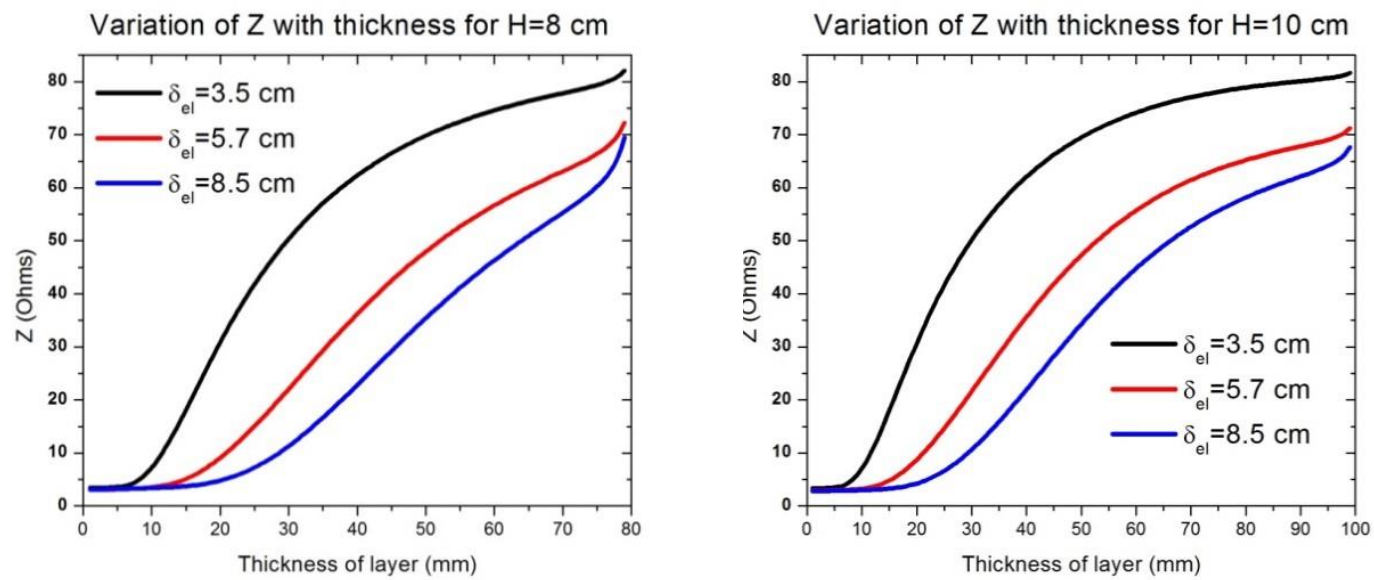

Figure-4: Variation of the FIM transfer impedance with thickness of resistive layer for a box height $\mathrm{H}=8 \mathrm{~cm}$ (left) and $10 \mathrm{~cm}$ (right) respectively. The series are for electrode separations of $3.5,5.7$ and $8.5 \mathrm{~cm}$ respectively. The non-linearity of the plots increases and the second knee almost disappears for greater box heights.

We also studied these behaviours for different box heights $H$ and the results are shown in Figure 4 for $\mathrm{H}=8 \mathrm{~cm}$ and $10 \mathrm{~cm}$, respectively. It can be seen that for a greater box height, the second knee is much less pronounced, and it almost disappears with further increase in box height. An increased nonlinearity may be noted in the impedance plots for boxes with larger heights for the same electrode separations.

\section{(b) Variation of Transfer Impedance with Electrode Separation:}

Figure 5a represents the FIM value with respect to electrode separation for a box height $H=12 \mathrm{~cm}$, for different thicknesses of the resistive layers. It can be seen that as the separation between the 
electrodes is increased, the transfer impedance decreases. At large electrode separations, the transfer impedance tends to reach an asymptotic flat value, independent of the thickness of the resistive layer, which has been tried up to a thickness of $25 \mathrm{~mm}$. From an intuitive standpoint, it may be argued that the plot may not reach the above asymptotic value for greater thicknesses of the resistive layer.
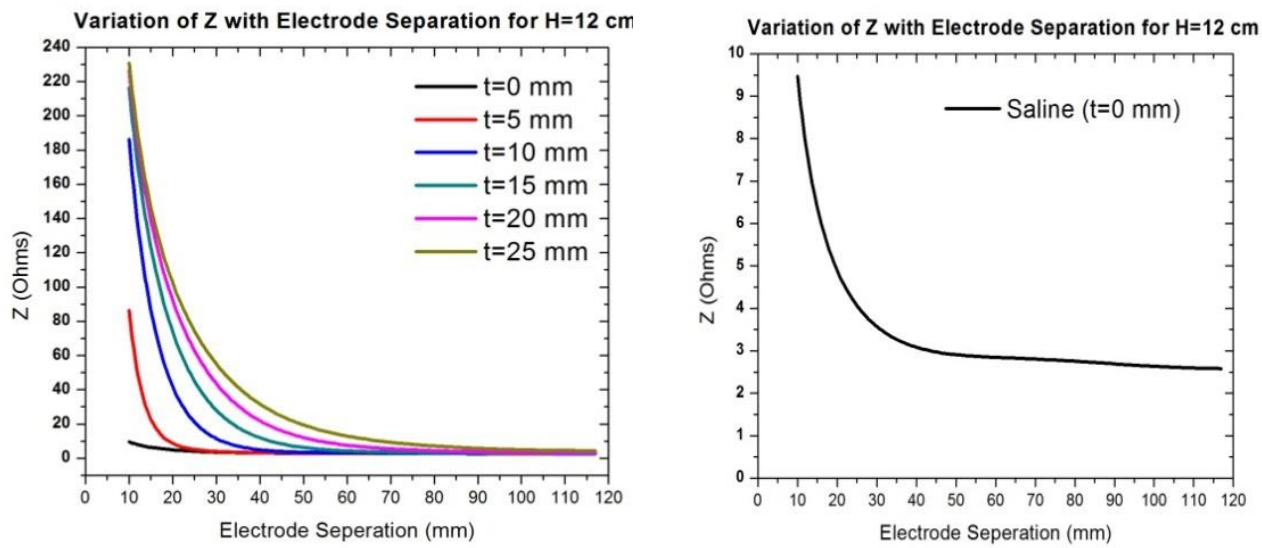

Figure 5: a) Variation of transfer impedance in FIM-4 with respect to electrode separation for different thicknesses of the simulated fat layers. For large electrode separation, $\mathrm{Z}$ reaches an asymptotic value, independent of $\mathrm{t}$ up to about $\mathrm{t}=25 \mathrm{~mm}$. b). The same curve for $\mathrm{t}=0$ shown in an expanded vertical scale.

The variation in the curve without any resistive layer ( $t=0$, background saline only) is very small compared to these for the simulated non-zero $t$ values $(5 \mathrm{~mm}$ to $25 \mathrm{~mm})$. Therefore it has been shown separately in Figure $5 \mathrm{~b}$ with an expanded vertical scale. It is seen to follow the same decreasing trend reaching again a small asymptotic value.

In order to isolate the effect of the thickness of the resistive layer only, we may plot the normalized transfer impedance $\left(Z_{N}\right)$ obtained by dividing the impedance values by the corresponding value of the saline material only, at each electrode separation. These are shown in Figure $6(a, b, c)$ for box heights of $4 \mathrm{~cm}, 6 \mathrm{~cm}$ and $12 \mathrm{~cm}$, respectively. The normalized impedance decreases rapidly at first with the increase of electrode separation, moves through a point of maximum curvature, and then reaches an asymptotic value which is slightly different for different thicknesses of the resistive layer. It may be noted that the asymptotic value of the normalized impedance is constant, for each thickness of the resistive layer, implying that most of the contribution to the impedance comes from the flow of current through the saline. Hence the value is close to unity, but not exactly equal to 1 .

Notice that the normalized impedance slightly decreases with the increase of box height (cf Figures $6 \mathrm{a}, 6 \mathrm{~b}$ and $6 \mathrm{c}$ ) for the same thickness $\mathrm{t}$ and at same electrode separation. This arises from the change of impedance with box height as discussed in the next section. 


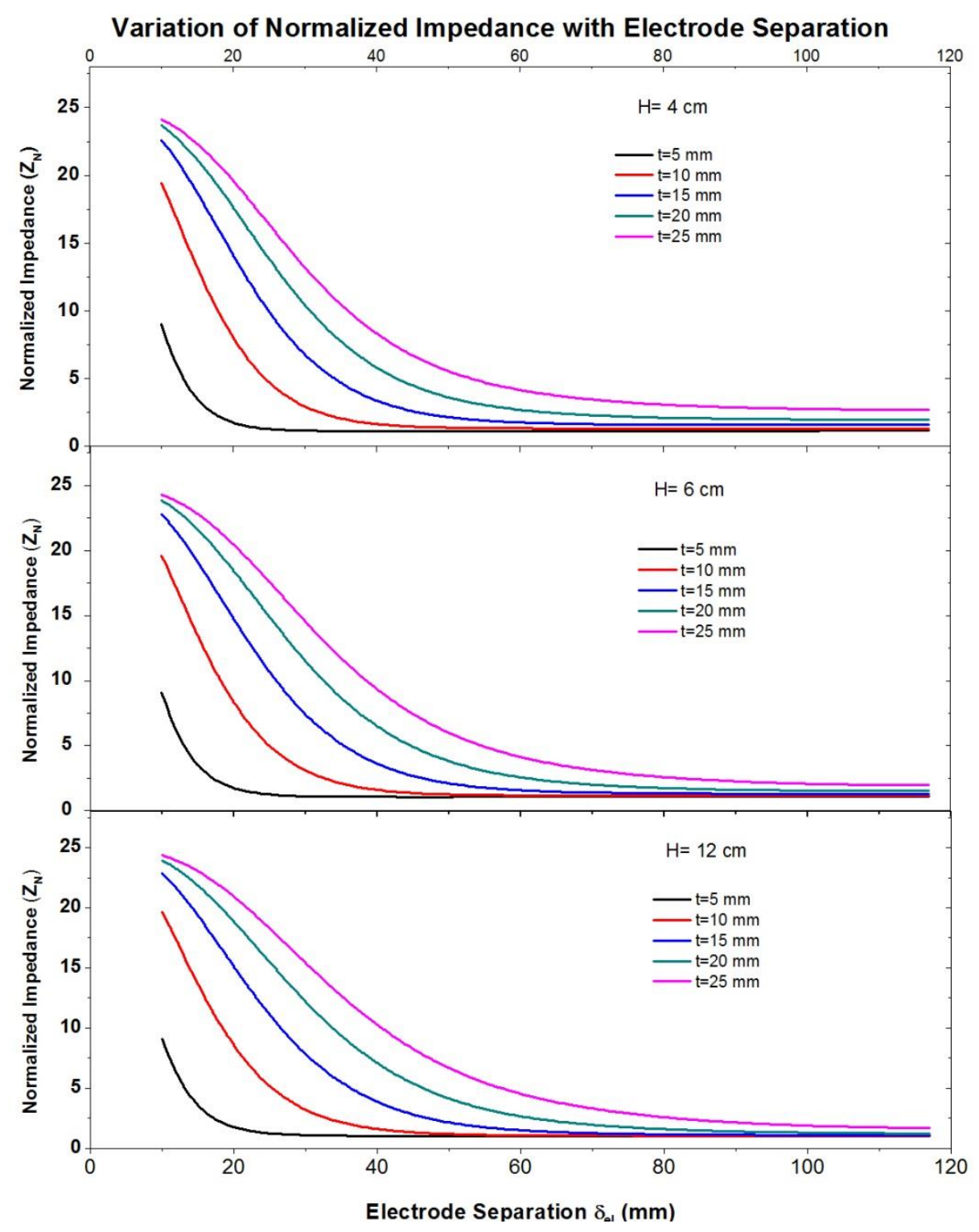

Figure-6(a,b,c): Variation of normalized impedance with electrode separation. The maximum of the normalized impedance are of the same order and all reaches a small asymptotic value of 1 . Figure (a,b,c) corresponds to box height of $4 \mathrm{~cm}, 6 \mathrm{~cm}$ and 12 $\mathrm{cm}$, respectively.

(c) Variation of Transfer Impedance with Height of the Box:

For small electrode separation the values of the transfer impedance $(Z)$ are almost independent of the box height, for the same thickness of the resistive layer and electrode separation. At large electrode separation, $\left(\delta_{e l}=8.5 \mathrm{~cm}\right), Z$ decreases slightly with box height. This is seen in Figure 7 . 


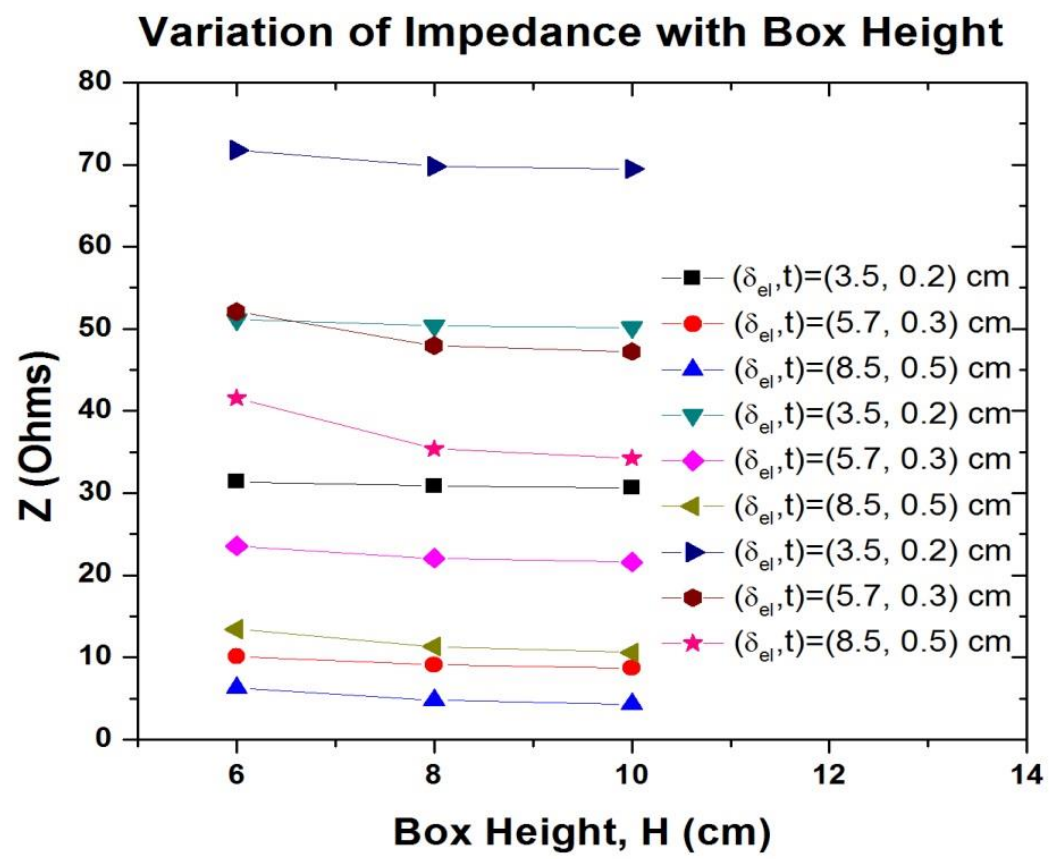

Figure 7: Variation of transfer impedance $Z$ for the same thickness of resistive layer and electrode separation for different box heights $(\mathrm{H})$. At small electrode separation, the effect of box height is almost negligible while at larger electrode separation, impedance slightly decreases with $\mathrm{H}$.

In order to further isolate the effect of the height of the box, we kept the other parameters fixed and simulated the model to find out the variation of the transfer impedance. To this end, we have calculated the transfer impedance for the background saline material varying the electrode separation. The results shown in figure 8 shows some interesting finite-size-effect of the height of the box. For small values of the electrode separation, the transfer impedance decreases rapidly with the increase of the electrode separation, reaches a minimum. And then increases to a saturation value, which differs slightly for different box heights. However, at the initial rapidly decreasing region, the impedance is almost independent of the box height. For larger values of the box height the minimum is less pronounced or absent and the transfer impedance reaches saturation values which are different slightly for different box heights.

\section{(d) Variation of the Transfer Impedance with Length/Width of the Box:}

It may be noted that, we are using a box of width and length of $12 \mathrm{~cm}$ each. The cylindrical electrodes have a diameter of $0.25 \mathrm{~cm}$ and hence they cannot be put at a separation more than $117.5 \mathrm{~mm}(120-$ $2 \times(1.25))$. The maximum separation we have used is $117 \mathrm{~mm}$, which is very close to the allowed limit. As seen in figures $5(\mathrm{a}, \mathrm{b})$ and $6(\mathrm{a}, \mathrm{b}, \mathrm{c})$, the transfer impedance does not show any noticeable variation at this limiting value, at large electrode separations. In all these figures, the transfer impedance almost reaches asymptotic or saturation values. This implies that very little current flows farther away from the electrodes and is being impeded by the non-conducting walls of the box. 


\section{Variation of ZN with Electrode Separration for Saline}

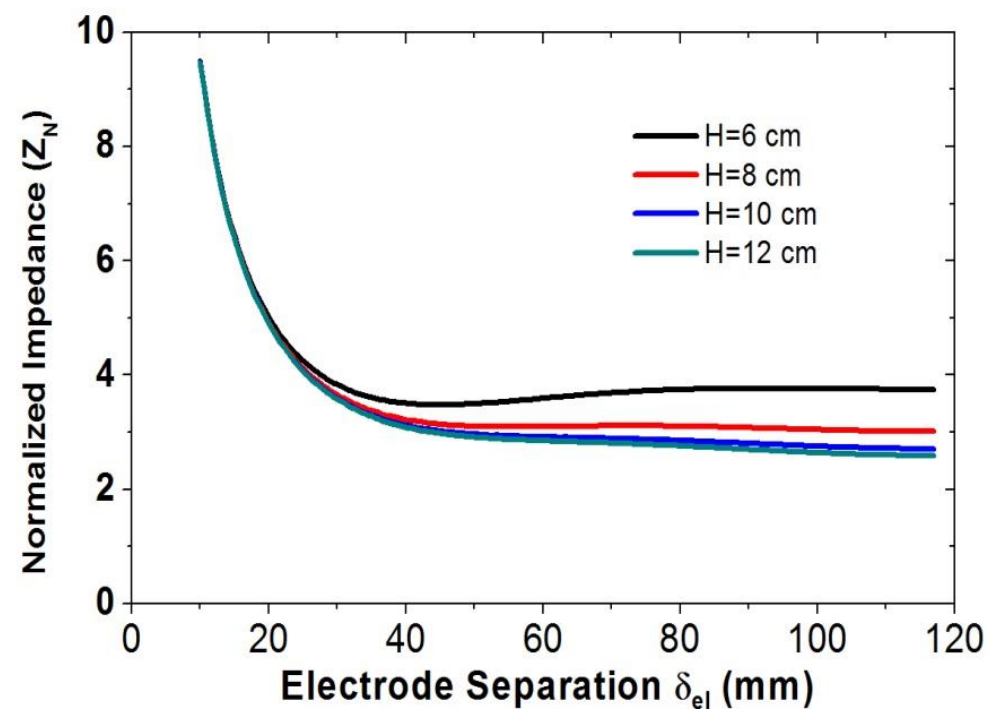

Figure 8: Variation of transfer impedance with electrode separation for background saline material only. For smaller heights of the box, the impedance plot has a minimum and then increases to saturation value.

\section{Explanations of Results}

In this section an attempt has been made to explain the trends in the transfer impedance demonstrated by the above mentioned results. These may be explained considering the current and electric potential distributions inside the box as the thickness, electrode separation, height and length/width of the box are varied.

(a) Variation of Transfer Impedance with Thickness of Resistive Layer: The behaviour of the transfer impedance in figures 3 and 4 may be explained if we consider the current paths within the resistive layers and further inside the saline, as shown in the figures 9(a) and 9(b), respectively.

For small thicknesses of the resistive layer, very little of the current flows within the thin layer, while most of it flows within the saline above (Fig 9a), the depth of which varies little. Therefore, the impedance is almost constant with an increase of the thickness of the resistive layer. For larger electrode separations, this effect extends further in respect of the thickness of the resistive layer, which is also observed in these figures.

As the thickness increases past the first knee, in figures 3 and 4, the length of the current paths no longer remain the same as shown in Figure-9(a) and becomes more like that shown in Figure-9(b), with more fraction of the current passing through the resistive layer. Therefore, the resistive layer contributes more to the transfer impedance, resulting in an increase of the transfer impedance with thickness. Again, reducing the thickness of the saline layer will also contribute to an increase of the transfer impedance. Due to these two effects, the variation of the impedance with thickness of the resistive layer is rather complex and non-linear.

At the later part of the plot, the impedance increases sharply for all three electrode separations. We have a second "knee" of the curves. In this region, the percentage change in the thickness of the resistive layer is very small, while that for the saline layer would be very high. Therefore, this sharp rise in transfer impedance may be attributed to the reduction of the depth of the saline layers. In all the cases, the sudden change happens in the last few centimeters of the saline layer thickness. 
Another point worth noting is the curvature of the graphs in the middle region. For small electrode separations, the curves are non-linear for all the three box heights chosen. The non-linearity decreases (i.e. goes towards linearity) as the electrode separation is increased. It may be noticed that at the thickness where the second knee occurs, the impedance value is larger for smaller electrode separation than for larger separations. Furthermore the second knee occurs at a slightly smaller thickness for larger electrode separations.

At a fixed thickness (Fig. 5-8) of the resistive layer, if the electrode separation is small, most of the current passes through the resistive layer. With increased electrode separation, more fraction of the current flows beyond the resistive layer into the saline volume, where the resistance is low.

Therefore, the transfer impedance decreases with increased electrode separation which has been seen. As the thickness of the resistive layer increases, more and more current paths will enter into the

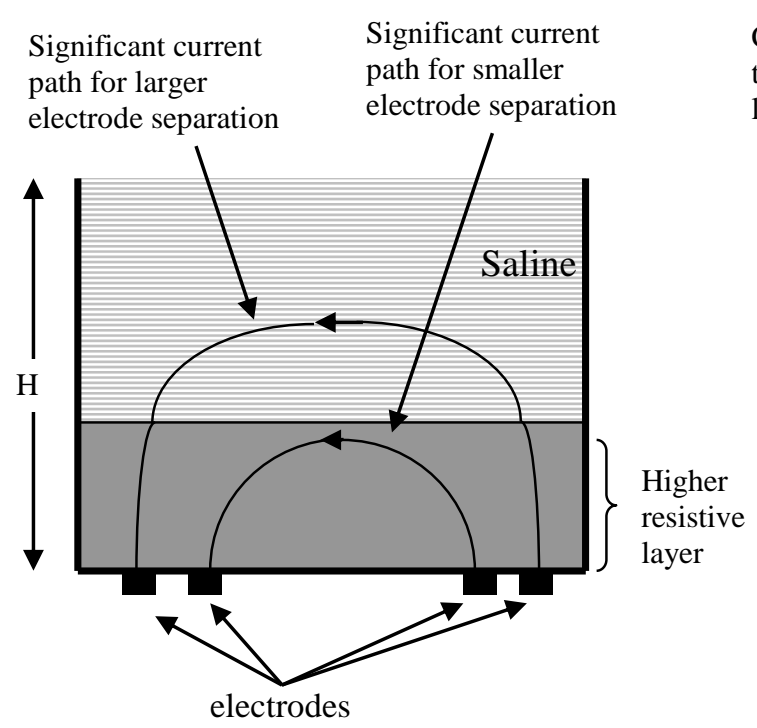

Figure 9(a): Current paths in saline-resistive layer system with different electrode separations

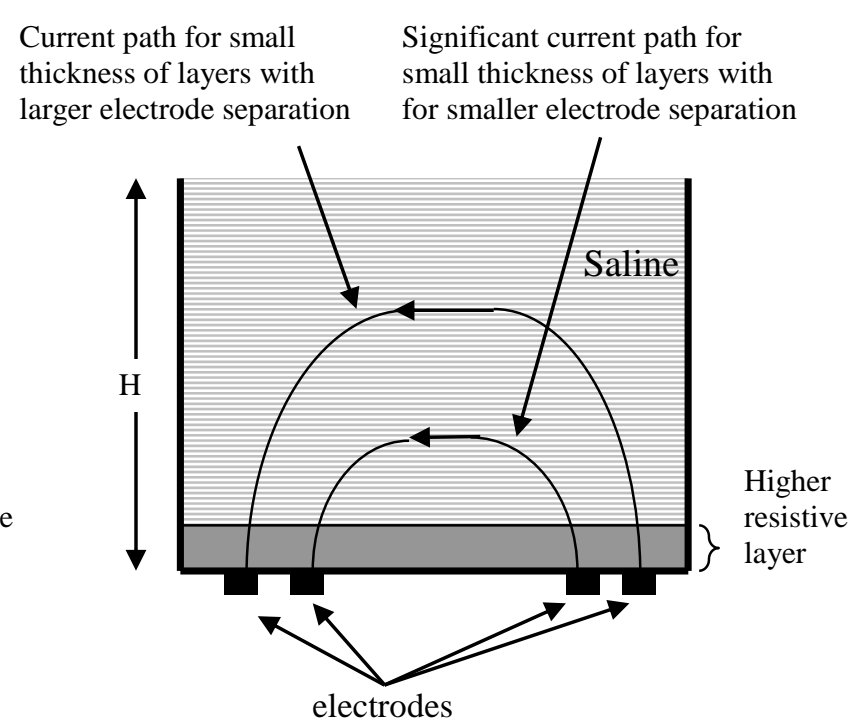

Figure 9(b): Current paths for less thick resistive layers in saline-resistive layer system

resistive layer from the less resistive saline above. This will create two opposing effects: (a) As more fraction of the input current flows through the resistive layer, potential drop between the receive electrodes will increase resulting in larger transfer impedance. (b) On the other hand, as the thickness of the resistive layer increases, wider cross-sectional area will be available for the current to flow through the resistive layers. This will tend to decrease the transfer impedance. The combined effect of the two will result in a non-trivial and non-linear increase of the impedance, (especially for smaller electrode separation) with the increase of the thickness. For electrode separation of $3.5 \mathrm{~cm}$ the initial increase of the impedance is dominated by the first effect up to a thickness of $\sim 35 \mathrm{~mm}$ when the slope of the impedance plot decreases, resulting in a point of maximum curvature. For larger electrode separation of $5.7 \mathrm{~cm}$ the point of maximum curvature is not so prominent and the increasing effect continues up to larger thickness. For electrode separation of $8.5 \mathrm{~cm}$, the point of maximum curvature is totally absent.

As the box height increases, the second knee almost disappears and the non-linearity of the graphs increases, as seen in the Figure 4. This can be understood as follows: For higher box height, even with larger electrode separation, there is scope of similar shaped current paths at larger thickness of the 
resistive layers. Hence we can conclude that the second knee thickness is a finite-size-effect due to the height of the box.

\section{(b) Variation of Transfer Impedance with Electrode Separation:}

The transfer impedance initially falls sharply with increase of the electrode separation going through a point of maximum curvature and then asymptotically becomes constant to a very low but positive value (Figure-5). This may be explained in terms of an increase of volume for current path which contributes to a decrease of impedance.

Point of Maximum Curvature for Thickness Determination Calibration Curve: From figure 6 and 7, we first obtained the points of maximum curvature for each curve and then the corresponding electrode separation. These electrode separations are then plotted against the thickness of the resistive layer in Figure 10.

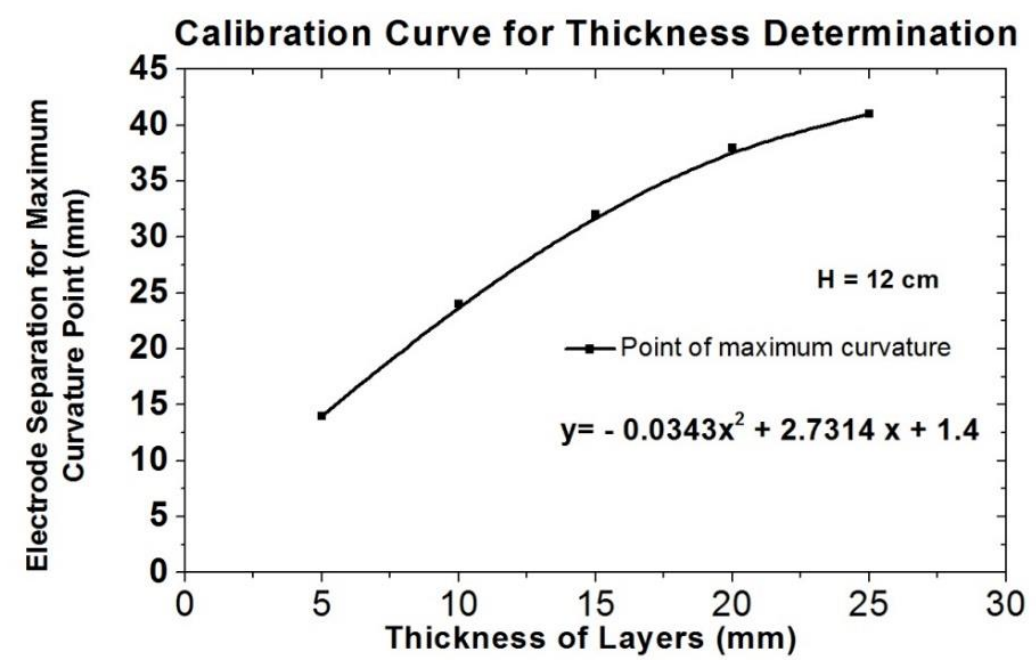

Figure 10: Variation of the electrode separation for points of maximum curvature with the thickness of the resistive layer, $\mathrm{H}=12 \mathrm{~cm}$.

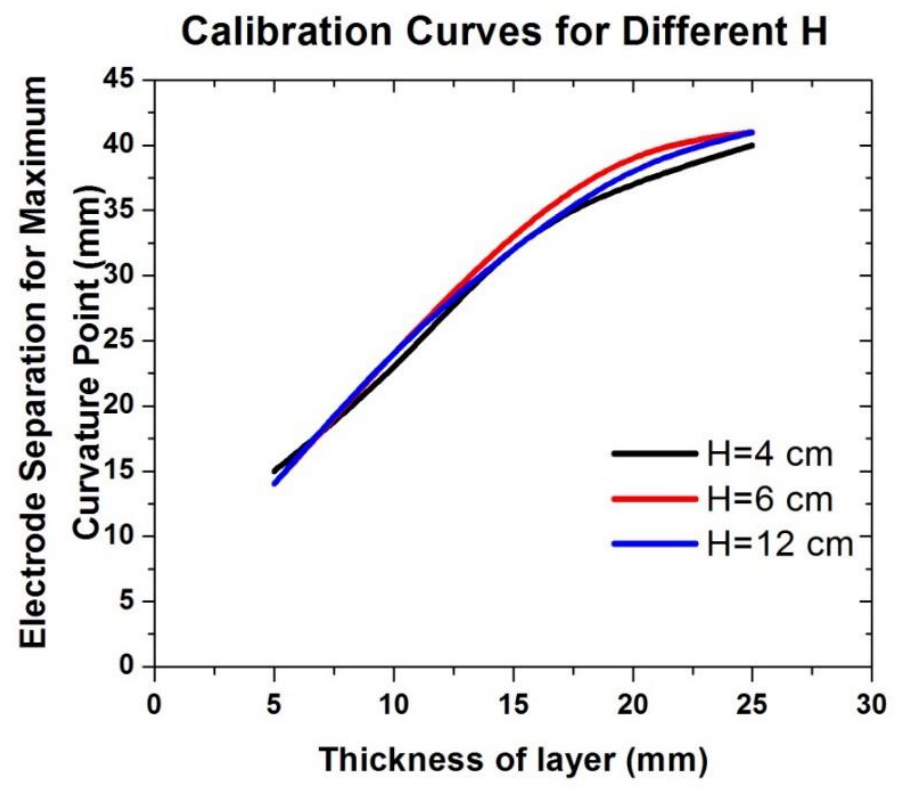

Figure 11: Calibration curves for determination of thickness of the resistive layers for different heights of the box. 
As the thickness of the resistive layer increases, the point of maximum curvature occurs at larger electrode separation. This curve appears to be unique for a particular box height and may be used as a calibration curve for the determination of the thickness. However, Figure 11 shows that the calibration curve varies very little with box height.

\section{(c) Variation of Transfer Impedance with Height of the Box:}

As seen in figure 8 , for small values of the box height, the transfer impedance at first decreases with the increase of the electrode separation, reaches a minimum and then increases to a saturation value. This can be explained from the picture of the current path in Fig 9. As the electrode separation increases, more and more fraction of the current reaches higher heights of the box. If the box is not sufficiently high, at a particular electrode separation, currents paths will be impeded by the upper boundary of the box resulting in a subsequent increase of the transfer impedance. As the electrode separation further increases, the current paths are squeezed but do not substantially increase the impedance, hence reaches a saturation value. For larger box heights, the fraction of current becomes progressively smaller at higher heights (z-value) of the box. Hence this dip in the impedance is seen to diminish and we have almost monotonic decrease of the transfer impedance.

\section{DISCUSSIONS}

In the present simulation work, we have used a rectangular box with different heights and thickness of the boundary resistive layer. The shape of box, although taken to be of a particular type (rectangular), the results we get can be applied at least qualitatively for any finite sized enclosure of any shape. This is because we have investigated effects of the lateral dimensions (length or width) and that of the height and have found only the height to affect the impedance significantly.

The choice of the saline was dictated by larger conductivity value (Weast, ed, 1989, Nörtemann et al., 1997), and scope of comparison with phantom studies. The material characteristics of the simulated fat layer were taken to be that of the fat as found in the literature (Gabriel et al. 1996, Bronzino ed. 2000). Since we were looking for a method of determination of the thickness, which is a geometrical characteristics of the setup, using any suitable electrical parameter values will suffice. Any variation of the permittivity or electrical conductivity will not change the characteristic trends of the curves, and at best can change the position or the slopes. This justifies our choice of material parameters values.

All the models use alternating current of (10 kHz frequency), which is much appropriate for the biological tissues or materials having complex impedance. Our calculated values were the absolute value of the transfer impedance, which may be complex in general. At the chosen frequency if a small current of the order $\mathrm{mA}$ is used, it is safe for the human body. Although our method is general enough to be used in industrial applications, we expect it to be primarily used in bio-impedance applications.

Previous work by Surovy et al. (2012) used the variation of the slope of the impedance versus electrode separation curve as a means to determine the thickness of resistive fat layer. In that work, only three sets of electrodes were used and it was evident that not all electrode separation could be used for all thickness or type of fat. To investigate this we varied the electrode separation over a wide range and indeed found that the most prominent feature may occur at larger electrode separations than 
that considered by Surovy et al. (2012). This justifies the simulation study as it extended the previous work.

Furthermore, instead of using a calibration curve for the slope versus thickness, it is more appropriate to use the unique features of the impedance curves, the point of maximum curvature, which is a single point in the curve relating to a length. The transfer impedance will in general depend on the material properties, frequency, electrode separation, thickness of the layer and height of the box, i.e. $Z=$ $Z\left(\{\epsilon, \sigma, \omega\},, \delta_{e l}, t, H\right)$. However, the effect of the box height is seen to diminish at larger heights of the box. Furthermore, to remove any residual/remaining effect, we have used the normalized impedance $Z_{N}=Z / Z_{S}$, and fixed material parameters. This makes the normalized impedance a function of only two length scales, the electrode separation and the thickness of the resistive layer:

$$
Z_{N}=Z_{N}\left(\left\{\epsilon_{i}\right\}, \delta_{e l}, t\right)
$$

In an impedance curve of fixed thickness, since the point of maximum curvature is a unique point, it must be related somehow to the thickness (the electrode separation is a variable here). This motivates us to use the position of the point of maximum curvature i.e. the corresponding electrode separation as a function of the thickness and obtain a calibration curve.

Similar point of maximum curvature is seen in the impedance versus thickness curve for fixed electrode separations, but these cannot be used as calibration curve as obtaining such a curve is not practically possible (by varying thickness of fat layer).

\section{CONCLUSIONS}

We have devised a very useful method for the determination of the thickness of a resistive boundary layer in a volume conductor using FIM. This is an attractive application of FIM and provides a simple yet effective tool for medical purposes. This work may be extended by varying the electrical properties of the materials used, for enclosures of more realistic shape or for multi-frequency case. More than one layer of resistive materials may also be used in future works. The present work will provide guidance for further extensions.

\section{ACKNOWLEDGEMENTS}

The authors gratefully acknowledge the support from the International Science Programme (ISP), Uppsala University, Sweden in procuring a license for COMSOL and for subsistence support to some of the authors.

\section{REFERENCES}

Birgersson UH, Birgersson E and Ollmar S, 2012, Estimating electrical properties and the thickness of skin with electrical impedance spectroscopy: Mathematical analysis and measurements. Journal of Electrical Bioimpedance, 3(1), 51-60, DOI: http://dx.doi.org/10.5617/jeb.400 .

Bronzino JD, Ed. 2000, Values of Specific Conductance for Human Tissues, Biomedical Engineering Handbook, 2, 68-2, CRC press, Chicago.

Brown BH, Barber DC, Morice AH, and Leathard AD,1994, Cardiac and respiratory related electrical impedance changes in the human thorax. Biomedical Engineering, IEEE Transactions on, 41, 729-734. DOI: $10.1109 / 10.310088$.

Gabriel C, Gabriel S and Corthout E, 1996, The dielectric properties of biological tissues: I. Literature survey. Physics in Medicine and Biology, 41, 2231, http://iopscience.iop.org/0031$\underline{9155 / 41 / 11 / 001}$. 
Haowlader S, Baig TN and Rabbani KS, 2010, Abdominal fat thickness measurement using Focused Impedance Method (FIM) - phantom study, Journal of Physics: Conference Series, 224, 012061, DOI: $10.1088 / 1742-6596 / 224 / 1 / 012061$.

Islam N, Rabbani KSE and Wilson A, 2010, The sensitivity of focused electrical impedance measurements. Physiological Measurement, 31, S97-S109, http://iopscience.iop.org/09673334/31/8/S08

Kadir MA, Ahmed SP, Al-Quaderi GD, Rahman R and Rabbani KS, 2013, Application of Focused Impedance Method (FIM) to Determine the Volume of an Object within a Volume Conductor, Proceedings of the 2013 COMSOL Conference in Bangalore, https://www.comsol.com/paper/download/182751/kadir_paper.pdf .

Nörtemann K, Hilland J and Kaatze U, 1997, Dielectric properties of aqueous $\mathrm{NaCl}$ solutions at microwave frequencies, The Journal of Physical Chemistry A, 101, 6864-6869, http://pubs.acs.org/doi/abs/10.1021/jp971623a .

Pethig R, 1979, Dielectric and Electronic properties of Biological materials, New York, John Wiley, ISBN-13: 978-0471997283.

Scharfetter H, Schlager T, Stollberger R, Felsberger R, Hutten H and Hinghofer-Szalkay H, 2001, Assessing abdominal fatness with local bioimpedance analysis: basics and experimental findings, International Journal of Obesity, 25, 502-511 http://www.nature.com/ijo/journal/v25/n4/pdf/0801556a.pdf .

Schwan HP 1957, Electrical properties of tissue and cell suspensions. Advances in biological and medical physics, JH Lawrence and CA Tobias, Eds, 5, 147-209.

Smulders L A W and van Oosterom A (1992). Application of electrical impedance tomography to the determination of lung volume, Clinical Physics and Physiological Measurement, 13(A), 167170, http://iopscience.iop.org/0143-0815/13/A/032 .

Surovy NJ, Billah MM, Haowlader S Al-Quaderi GD and Rabbani KS, 2012, Determination of abdominal fat thickness using dual electrode separation in the focused impedance method (FIM). Physiological Measurement, 33, 707-718, DOI:10.1088/0967-3334/33/5/707 .

Rabbani KS and Karal MAS, 2008, A new four-electrode Focused Impedance Measurement (FIM) system for physiological study. Annals of biomedical engineering, 36, 1072-1077, http://link.springer.com/article/10.1007/s10439-008-9470-7\#page-1.

Rabbani KS, Sarker M, Akond MHR and Akter T, 1999, Focused impedance measurement (FIM): a new technique with improved zone localization. Annals of the New York Academy of Sciences, 873, 408-420, http://onlinelibrary.wiley.com/doi/10.1111/j.17496632.1999.tb09490.x/full .

Weast RC, Lide DR, Astle MJ and Beyer WH, Eds, 1989, CRC Handbook of Chemistry and Physics, D-221, CRC Press, Boca Raton, FL.

Weinstein L, 2003, Electrical-Impedance-Based Ice-Thickness Gauges. NASA Tech Briefs, p-8, http://ntrs.nasa.gov/archive/nasa/casi.ntrs.nasa.gov/20110023969.pdf . 